\title{
miR-576-3p overexpression enhances cisplatin sensitivity of ovarian cancer cells by dysregulating PD-L1 and cyclin D1
}

\author{
YING ZUO $^{1 *}$, WEI ZHENG ${ }^{1 *}$, QING TANG ${ }^{1}$, JING LIU $^{2}$, SHANSHAN WANG $^{1}$ and CHUNXIA XIN ${ }^{3}$ \\ Departments of ${ }^{1}$ Gynecology, ${ }^{2}$ Pathology and ${ }^{3}$ Medical Oncology, Affiliated Yantai Yuhuangding Hospital, \\ Medical College of Qingdao University, Yantai, Shandong 264000, P.R. China
}

Received February 3, 2020; Accepted August 14, 2020

DOI: $10.3892 / \mathrm{mmr} .2020 .11719$

\begin{abstract}
Cisplatin (DDP) resistance is a major obstacle in the chemotherapeutic efficacy of ovarian cancer. The present study aimed to explore the role of miR-576-3p in DDP sensitivity of ovarian cancer cells. Ovarian cancer cell lines SKOV3 and A2780 and DDP-resistant ovarian cancer cell lines SKOV3/DDP and A2780/DDP were used in the present study. In vitro studies demonstrated that microRNA (miR)-576-3p overexpression increased the DDP sensitivity of DDP-resistant ovarian cancer cells. A dual-luciferase assay verified that both programmed death-ligand 1 (PD-L1) and cyclin D1 were targets of miR-276-3p and were reversely associated with the expression of miR-576-3p. Moreover, in vivo studies indicated that tumorigenesis was inhibited by DDP, which was enhanced by further miR-576-3p overexpression in tumor tissues. Taken together, the results suggested that miR-576-3p overexpression increased DDP chemosensitivity of ovarian cancer cells via decreasing PD-L1 and cyclin D1, indicating that miR-576-3p may serve as a promising therapeutic target for ovarian cancer.
\end{abstract}

\section{Introduction}

Ovarian cancer is the second most commonly diagnosed and most deadly gynecological malignancy worldwide (1). According to 2018 statistics, 225,500 women were diagnosed with ovarian cancer, and 140,200 women died from this disease worldwide (2). Etiological studies reported that childbearing, family history of ovarian cancer, smoking, obesity, and diet may increase the risk of ovarian cancer (3). At present, the conventional treatment of ovarian cancer is cytoreductive

Correspondence to: Miss Chunxia Xin, Department of Medical Oncology, Affiliated Yantai Yuhuangding Hospital, Medical College of Qingdao University, 20 Yuhuangding East Road, Yantai, Shandong 264000, P.R. China

E-mail: chunxiaxin2019@163.com

${ }^{*}$ Contributed equally

Key words: chemosensitivity, cyclin D1, programmed death-ligand 1, cisplatin, microRNA-576-3p, ovarian cancer surgery, followed by chemotherapy and radiotherapy (4). Cisplatin (DDP) and paclitaxel are the main chemotherapeutic drugs used in ovarian cancer. However, $>60 \%$ of the patients develop advanced-stage relapse or do not respond to chemotherapy $(5,6)$. Although the majority of patients are initially sensitive to chemotherapy, they often develop resistance after multiple relapses. Due to the lack of effective second-line chemotherapy, the overall survival of patients is short, at only 1 year (7). Therefore, it is crucial to overcome the drug resistance of patients with ovarian cancer to improve their prognosis.

MicroRNAs (miRs/miRNAs) are small non-coding RNA molecules that regulate gene expression by silencing or degrading their target mRNA molecules, thereby mediating various pathological processes, including tumorigenesis (8). Various miRNAs are dysregulated in several cancers. Among them, miR-576-3p was found to be downregulated in non-melanoma skin cancer (9). In lung adenocarcinoma, miR-576-3p was significantly reduced, and its overexpression reduced the expression of mesenchymal markers, and inhibited the migration and invasion of lung adenocarcinoma cells (10). miR-576-3p downregulation was also observed in bladder cancer tissues and was found to be correlated with poor clinical outcome (11). These findings indicated the negative regulatory effect of miR-576-3p on cancer development. Moreover, miR-576-3p may also be closely associated with the chemosensitivity of tumors. Studies have found that miR-576-3p was downregulated in DDP-resistant human teratoma cells, as well as Adriamycin-resistant breast cancer tissues and cells $(12,13)$. However, further investigation is required to determine whether miR-576-3p plays an important role in mediating the sensitivity of ovarian cancer cells to DDP-based chemotherapy.

In present study, TargetScan7.2 (http://www.targetscan. org/vert_72/) predicted that miR-576-3p may target programmed death-ligand 1 (PD-L1) and cyclin D1. PD-L1 has been demonstrated to be associated with cancer prognosis and is highly expressed in gastric, colon, and lung cancers (14-16). PD-1/PD-L1 signal blockade is beneficial for the treatment of cancer, including colon and liver cancer $(15,17)$. Moreover, PD-L1 was found to be upregulated in DDP-resistant ovarian cancer cells, and downregulation of PD-L1 increased DDP sensitivity in lung cancer $(18,19)$. Cyclin D1 is a key regulator of the G1/S transition and has been reported to play 
a key role in tumor cell development and drug resistance (20). Downregulation of cyclin D1 increased DDP chemosensitivity in gastric cancer (21), whereas overexpression of cyclin D1 was revealed to promote tumor cell growth and resistance of pancreatic tumor cell lines to DDP-mediated apoptosis (22). Thus, based on the significant role of PD-L1 and cyclin D1 in cancer development and chemotherapy sensitivity, the present study investigated whether miR-576-3p affects the chemosensitivity of ovarian cancer cells to DDP by regulating PD-L1 and cyclin D1.

\section{Materials and methods}

Cell culture, transfection and drug administration. Cell culture, transfection, and treatment of human ovarian cancer cells were performed as described previously (19). Ovarian cancer cells SKOV3 and A2780 were purchased from Procell Life Science \& Technology Co., Ltd. 293T cells were purchased from Shanghai Zhongqiao Xinzhou Biotechnology Co., Ltd. DDP was purchased from Dalian Meilun Biology Technology Co., Ltd. Cells were maintained in Minimum Essential Medium (Invitrogen; Thermo Fisher Scientific, Inc.) supplemented with $10 \%$ fetal bovine serum (Sigma-Aldrich; Merck $\mathrm{KGaA}$ ) in a $37^{\circ} \mathrm{C}$ incubator containing $5 \% \mathrm{CO}_{2}$. The transfection of miR-576-3p mimic, negative control (NC) mimic, miR-576-3p inhibitor, and NC inhibitor (100 pmol) into cells (at $90 \%$ confluence) was performed using Lipofectamine ${ }^{\circledR}$ 2000 (Invitrogen; Thermo Fisher Scientific, Inc.), according to the manufacturer's instructions. At $24 \mathrm{~h}$ post-transfection, cells were used for subsequent experiments. The sequences of the mimics and inhibitors were as follows: miR-576-3p mimic forward, 5'-AAGAUGUGGAAAAAUUGGAAUC-3' and reverse, 5'-UUCCAAUUUUUCCACAUCUUUU-3'; NC mimic forward, 5'-UUCUCCGAACGUGUCACGUTT-3' and reverse, 5'-ACGUGACACGUUCGGAGAATT-3'; miR-576-3p inhibitor, 5'-GAUUCCAAUUUUUCCACAUCUU-3'; NC inhibitor, 5'-UUGUACUACACAAAAGUACUG-3'. The sequences were purchased from JTS Scientific Biological Technology Co., Ltd.

Construction of DDP-resistant ovarian cancer cell lines. DDP-resistant ovarian cancer cell lines SKOV3/DDP and A2780/DDP were established by culturing SKOV3 and A2780 cells with increasing concentrations of DDP. Briefly, SKOV3 and A2780 cells were resuscitated and cultured in an incubator. At $60 \%$ confluence, cells were treated with DDP at concentrations of $1,2,4$, and $8 \mu \mathrm{M}$. Once the cells were incubated for $48 \mathrm{~h}$, the medium was discarded and fresh medium was added. After 12 weeks, stable DDP-resistant SKOV3/DDP and A2780/DDP cells were successfully established and stored in liquid nitrogen.

Cytotoxicity assay. Cells were seeded into 96-well plates at a cell count of $5 \times 10^{3}$ per well. At $24 \mathrm{~h}$ after transfection, medium $(200 \mu \mathrm{l})$ containing different concentrations of DPP $(5,10,20,40$, and $80 \mu \mathrm{M})$ was added to each well $(23,24)$. After the cells were incubated for $48 \mathrm{~h}$ at $37^{\circ} \mathrm{C}$ and $5 \% \mathrm{CO}_{2}$ MTT mixture $(0.5 \mathrm{mg} / \mathrm{ml})$ was added into each well, and cells were then incubated at $37^{\circ} \mathrm{C}$ for $5 \mathrm{~h}$. Subsequently, DMSO solution $(150 \mu \mathrm{l})$ was added to dissolve the purple formazan.
After 10 min of incubation in the dark, the optical density value of cells at a wavelength of $570 \mathrm{~nm}$ was measured and the half-maximal inhibitory concentration $\left(\mathrm{IC}_{50}\right)$ was calculated.

Reverse transcription-quantitative PCR (RT-qPCR) analysis. Total RNA was extracted from tissues or cells using a total RNA extraction kit (Tiangen Biotech Co., Ltd.) according to the manufacturer's instructions. The RNA concentration in each sample was detected by ultraviolet spectrophotometry (NanoDrop 2000; Thermo Fisher Scientific, Inc.). cDNA was reverse-transcribed from RNA using RNase inhibitor (BioTeke Corporation) and Super M-MLV Reverse Transcriptase (BioTeke Corporation). The following temperature protocol was used for reverse transcription for miR-576-3p: $37^{\circ} \mathrm{C}$ for $30 \mathrm{~min}, 42^{\circ} \mathrm{C}$ for $30 \mathrm{~min}$ and $70^{\circ} \mathrm{C}$ for $10 \mathrm{~min}$. The following temperature protocol was used for reverse transcription for cyclin D1 and PD-L1: $25^{\circ} \mathrm{C}$ for $10 \mathrm{~min}, 42^{\circ} \mathrm{C}$ for $50 \mathrm{~min}$ and $80^{\circ} \mathrm{C}$ for $10 \mathrm{~min}$. Subsequently, RT-qPCR analysis was performed with primers, SYBR-Green (Sigma-Aldrich; Merck KGaA) and 2X Power Taq PCR Master Mix (BioTeke Corporation) to detect miR-576-3p expression level. The following thermocycling conditions were used for qPCR for miR-576-3p: $94^{\circ} \mathrm{C}$ for $4 \mathrm{~min}, 40$ cycles of $94^{\circ} \mathrm{C}$ for $15 \mathrm{sec}, 60^{\circ} \mathrm{C}$ for $20 \mathrm{sec}$ and $72^{\circ} \mathrm{C}$ for $15 \mathrm{sec}$. The following thermocycling conditions were used for qPCR for cyclin D1 and PD-L1: $94^{\circ} \mathrm{C}$ for $5 \mathrm{~min}, 40$ cycles of $94^{\circ} \mathrm{C}$ for $15 \mathrm{sec}, 60^{\circ} \mathrm{C}$ for $25 \mathrm{sec}$ and $72^{\circ} \mathrm{C}$ for $30 \mathrm{sec}$. The primers used for qPCR were as follows: miR-576-3p forward, 5'-AAGATGTGGAAA AATTGG AATC-3' and reverse, 5'-GCAGGGTCCGAGGTATTC-3'; 5S rRNA forward, 5'-GATCTCGGAAGCTAAGCAGG-3' and reverse, 5'-TGGTGCAGGGTCCGAGGTAT-3'; cyclin D1 forward, 5'-GCGAGGAACAGAAGTGCG-3' and reverse, 5'-TGGAGTTGTCGGTGTAGATGC-3'; PD-L1 forward, 5'-AACTACCTCTGGCACATC-3' and reverse, 5'-ATCCAT CATTCTCCCTTT-3'; $\beta$-actin forward, 5'-GGCACCCAGCAC AATGAA-3' and reverse, 5'-TAGAAGCATTTGCGGTGG-3'. The primers were purchased from GenScript. The expression of miR-576-3p, and cyclin D1 and PD-L1 was calculated and normalized to 5S rRNA and $\beta$-actin, respectively. Gene expression was quantified using the $2^{-\Delta \Delta C q}$ method (25).

Western blot analysis. Total protein was extracted from tissues or cells using IP cell lysis buffer (Beyotime Institute of Biotechnology) and phenylmethylsulphonyl fluoride protease inhibitor (Beyotime Institute of Biotechnology). Protein concentration was quantified using a BCA protein assay kit (Beyotime Institute of Biotechnology), according to the manufacturer's protocol. Proteins $(20 \mu \mathrm{l} / \mathrm{lane})$ from each sample were separated via SDS-PAGE (8 and 13\% separation gel for different sized proteins) and then transferred to a PVDF membrane (EMD Millipore). After blocking in 5\% skimmed milk powder for $1 \mathrm{~h}$ at room temperature the membranes were incubated with primary antibodies overnight at $4{ }^{\circ} \mathrm{C}$, followed by incubation with horseradish peroxidase-conjugated anti-rabbit/mouse secondary antibodies for $2 \mathrm{~h}$ at room temperature (1:5,000; cat. nos. A0208 and A0216; Beyotime Institute of Biotechnology). The primary antibodies used were as follows: PD-L1 (1:1,000; cat.no.DF6526; Affinity Biosciences), cyclin D1 (1:1,000; cat. no. AF0931; Affinity Biosciences), efflux pump multidrug resistance protein 1 (MDR1; 1:1,000; 
cat. no. AF5185; Affinity Biosciences), pro/cleaved caspase-3 (1:1,000; cat. no. DF6879; Affinity Biosciences), pro/cleaved poly (ADP-ribose) polymerase (PARP; 1:1,000; cat. no. \#9532; Cell Signaling Technology, Inc.), and $\beta$-actin $(1: 1,000$; cat. no. sc-47778; Santa Cruz Biotechnology, Inc.). Finally, the membranes were visualized with ECL luminescent reagent (Beyotime Institute of Biotechnology) and the optical density values of the target bands were analyzed using Gel-Pro-Analyzer software (version 4; Media Cybernetics, Inc.). $\beta$-actin served as an internal control.

Cell cycle assay. Cells were incubated in 6-well plates at a density of $2 \times 10^{5}$ cells/well. After transfection with miR-576-3p mimic or NC mimic, cells were treated with DDP at $50 \%$ of the $\mathrm{IC}_{50}$ dose at $37^{\circ} \mathrm{C}$ for $24 \mathrm{~h}$. The treated cells were harvested and fixed in $70 \%$ ethanol at $4^{\circ} \mathrm{C}$ for $2 \mathrm{~h}$, followed by incubation with propidium iodide (PI; $25 \mu \mathrm{l})$ and RNase $\mathrm{A}(10 \mu \mathrm{l})$ at $37^{\circ} \mathrm{C}$ for $30 \mathrm{~min}$ in the dark. Finally, the cell cycle distribution was detected by flow cytometry using a NovoCyte flow cytometer (ACEA Biosciences, Inc.) and analyzed using NovoExpress software (version 1.2.5; ACEA Biosciences, Inc.).

Cell apoptosis assay. Both early and late apoptotic cells were assessed. Cells were seeded in 6-well plates at a density of $2 \times 10^{5}$ cells/well. After transfection, cells were treated with DDP at $50 \%$ of the $\mathrm{IC}_{50}$ dose at $37^{\circ} \mathrm{C}$ for $24 \mathrm{~h}$. Subsequently, cells were treated with $5 \mu \mathrm{l}$ Annexin V-fluorescein isothiocyanate (FITC) and $10 \mu \mathrm{l}$ PI for $20 \mathrm{~min}$ at room temperature in the dark. Finally, cells stained with V-FITC and PI were detected by flow cytometry using a NovoCyte flow cytometer (ACEA Biosciences, Inc.) and analyzed using NovoExpress (version 1.2.5; ACEA Biosciences, Inc.).

Dual-luciferase reporter assay. The binding sites between miR-576-3p and PD-L1 and cyclin D1 were determined using TargetScan (version 7.2; http://www.targetscan.org/vert_72/). PD-L1 and cyclin D1 fragments containing the target sequence of miR-576-3p were inserted into pmirGLO vectors (GenScript) to construct wild-type (wt) and mutant (mut) plasmids. Subsequently, the plasmids were co-transfected with miR-576-3p mimic, NC mimic, miR-576-3p inhibitor or NC inhibitor into $293 \mathrm{~T}$ cells (at $90 \%$ confluence) using Lipofectamine 2000 (Invitrogen; Thermo Fisher Scientific, Inc.). After $48 \mathrm{~h}$, luciferase activity was detected using a Dual-Luciferase Detection kit (Promega Corporation). Firefly luciferase activities were normalized to Renilla luciferase activities.

Animal experiments. The present study was approved by the Institutional Animal Care and Use Committee of Affiliated Yantai Yuhuangding Hospital (approval no. 2018137; Shandong, China) and performed according to the Guidelines for the Care and Use of Laboratory Animals (26). The feeding and treatment of mice were performed as described in our previous study (19). Briefly, 12 healthy female BALB/c nude mice (weight, $20 \mathrm{~g}$ ) aged 5-6 weeks were given free access to food and water and fed adaptively for 1 week. Mice were randomly divided into two groups: SKOV3/DDP + LV-NC + DDP group and SKOV3/DDP + LV-miR-576-3p + DDP group (n=6/group). The construction of SKOV3/DDP cells was performed as aforementioned. The nude mice were subcutaneously injected (the right side of the back of the neck) with lentivirus (LV) miR-576-3p infected SKOV3/DDP cells $\left(2 \times 10^{6}\right.$ in PBS) or LV control-infected SKOV3/DDP cells $\left(2 \times 10^{6}\right.$ in PBS). The tumor was confirmed by a pathologist. When the tumor volume reached $100 \mathrm{~mm}^{3}$, all nude mice were intraperitoneally injected with DDP $(10 \mathrm{mg} / \mathrm{kg})$ twice a week for 3 weeks. Tumor size was monitored every 2 days. After 19 days, nude mice were sacrificed by cervical dislocation. Death verification was confirmed by cessation of heartbeat and respiration, and absence of reflexes and metabolism. After the mice stopped breathing, the tumors were resected.

Statistical analysis. All data are presented as the mean \pm SD of three repeats, except for animal experiments $(n=6)$. GraphPad 8.0 (GraphPad Software, Inc.) was used to perform statistical analysis. An unpaired t-test, one-way ANOVA with Tukey's multiple comparisons test or two-way ANOVA with Sidak's multiple comparisons test were used to evaluate statistical significance. $\mathrm{P}<0.05$ was considered to indicate a statistically significant difference.

\section{Results}

miR-576-3p is downregulated whereas PD-L1 and cyclin $D 1$ are upregulated in DDP-resistant ovarian cancer cells. To investigate whether miR-576-3p, PD-L1 and cyclin D1 are involved in DDP resistance of ovarian cancer cells, DDP-resistant ovarian cancer cell lines SKOV3/DDP and A2780/DDP were used. The results demonstrated that, as the concentration of DDP increased, the viability of ovarian cancer cells SKOV3 and A2780 and DDP-resistant ovarian cancer cells SKOV3/DDP and A2780/DDP significantly decreased. Furthermore, the cell viability of ovarian cancer cells was more affected by DDP compared with that of DDP-resistant cells (Fig. 1A). In addition, the tolerance of SKOV3/DDP and A2780/DDP cells to DDP was significantly higher compared with that of SKOV3 and A2780 cells (Fig. 1B). RT-qPCR analysis was used to detect the mRNA expression of miR-576-3p in DDP-resistant cells, and the results revealed that the mRNA levels of miR-576-3p in SKOV3/DDP and A2780/DDP cells were downregulated compared with those in SKOV3 and A2780 cells (Fig. 1C). Western blotting demonstrated that the protein expression levels of PD-L1 and cyclin D1 in DDP-resistant cells were significantly higher compared with those in non-resistant cells (Fig. 1D and E). These data indicated that miR-576-3p, PD-L1 and cyclin D1 may be involved in DDP resistance of ovarian cancer cells.

miR-576-3p overexpression increases DDP sensitivity of DDP-resistant ovarian cancer cells in vitro. The role of miR-576-3p in ovarian cancer cells was investigated via transfection of miR-576-3p mimic, which significantly increased the expression of miR-576-3p in ovarian cancer cells (Fig. S1A). Furthermore, miR-576-3p overexpression decreased ovarian cancer cell viability, and promoted apoptosis and the accumulation of ovarian cancer cells in the G1 phase (Fig. S2). To study the effects of miR-576-3p on DDP sensitivity of ovarian cancer cells, DDP-resistant ovarian cancer cells were 

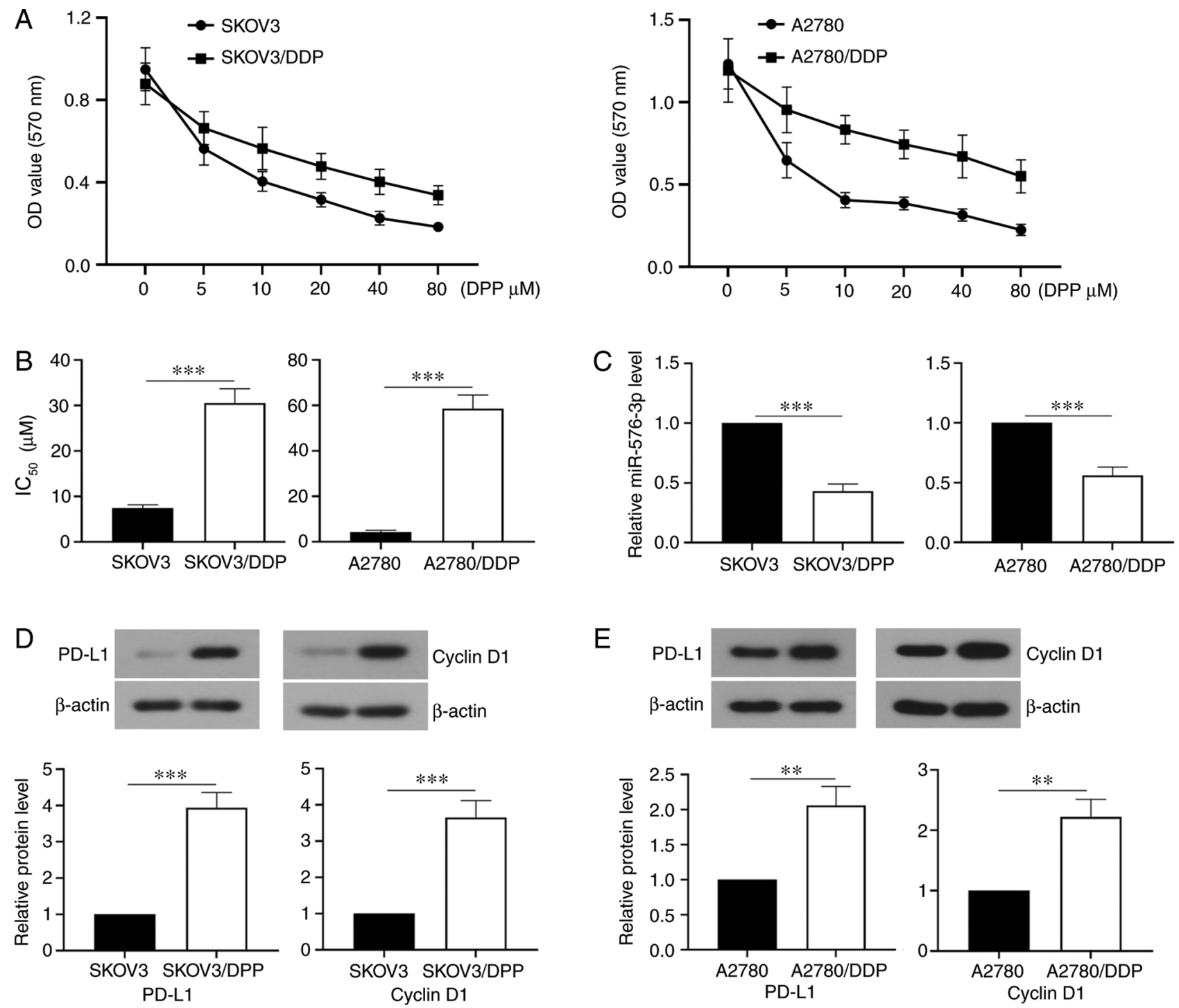

Figure 1. miR-576-3p is downregulated whereas PD-L1 and cyclin D1 is upregulated in DDP-resistant ovarian cancer cells. (A) Effects of DDP at increasing concentrations on the viability of ovarian cancer cells (SKOV3 and A2780) and DDP-resistant ovarian cancer cells (SKOV3/DDP and A2780/DDP). (B) IC 50 values of DDP in ovarian cancer cells and DDP-resistant ovarian cancer cells. (C) mRNA expression of miR-576-3p, and (D and E) protein expression levels of PD-L1 and cyclin D1 in each cell line. $\mathrm{n}=3 .{ }^{* *} \mathrm{P}<0.01$ and ${ }^{* * *} \mathrm{P}<0.001$. miR, microRNA; PD-L1, programmed death-ligand 1; DDP, cisplatin.

transfected with miR-576-3p mimic or NC mimic. The results demonstrated that miR-576-3p overexpression significantly increased miR-576-3p mRNA expression in DDP-resistant cells (Fig. 2A). miR-576-3p upregulation reduced DDP tolerance in DDP-resistant cells (Fig. 2B). Moreover, the viability of DDP-resistant cells decreased with the increase in DDP concentration, and miR-576-3p overexpression increased the DDP sensitivity of SKOV3/DDP and A2780/DDP cells (Fig. 2C). Furthermore, DDP treatment had no significant effect on DDP-resistant cell cycle progression and apoptosis compared with the control group (Fig. 2D and E). However, overexpression of miR-576-3p resulted in the accumulation of DDP-resistant cells in the G1 phase and promotion of cell apoptosis (Fig. 2D and E). Additionally, DDP treatment reduced the protein levels of PD-L1, cyclin D1 and MDR1, but increased the cleaved/pro caspase-3 ratio and cleaved/pro PARP ratio in DDP-treated DDP-resistant cells compared with the control group (Fig. 3). Moreover, miR-576-3p overexpression further enhanced the effects of DDP on PD-L1, cyclin D1,
MDR1, cleaved caspase-3 and cleaved PARP protein levels (Fig. 3). Therefore, miR-576-3p overexpression increased DDP sensitivity of DDP-resistant ovarian cancer cells via regulating cell viability and apoptosis.

miR-576-3p directly targets $P D-L 1$ and cyclin D1. A dual-luciferase assay was performed to detect the targeting relationship between miR-576-3p and PD-L1 and cyclin D1. The binding sites between miR-576-3p and PD-L1 and cyclin D1 were determined using TargetScan (version 7.2; http://www.targetscan.org/vert_72/). As shown in Fig. 4A and $\mathrm{B}$, the luciferase activity in cells transfected with wt-PD-L1 and wt-cyclin D1 plasmids was significantly decreased by miR-576-3p mimic transfection. The luciferase activity in cells transfected with mut-PD-L1 and mut-cyclin D1 plasmids showed no significant change. The results indicated that miR-576-3p can directly target PD-L1 and cyclin D1. Additionally, the mRNA and protein expression levels of PD-L1 and cyclin D1 were significantly 

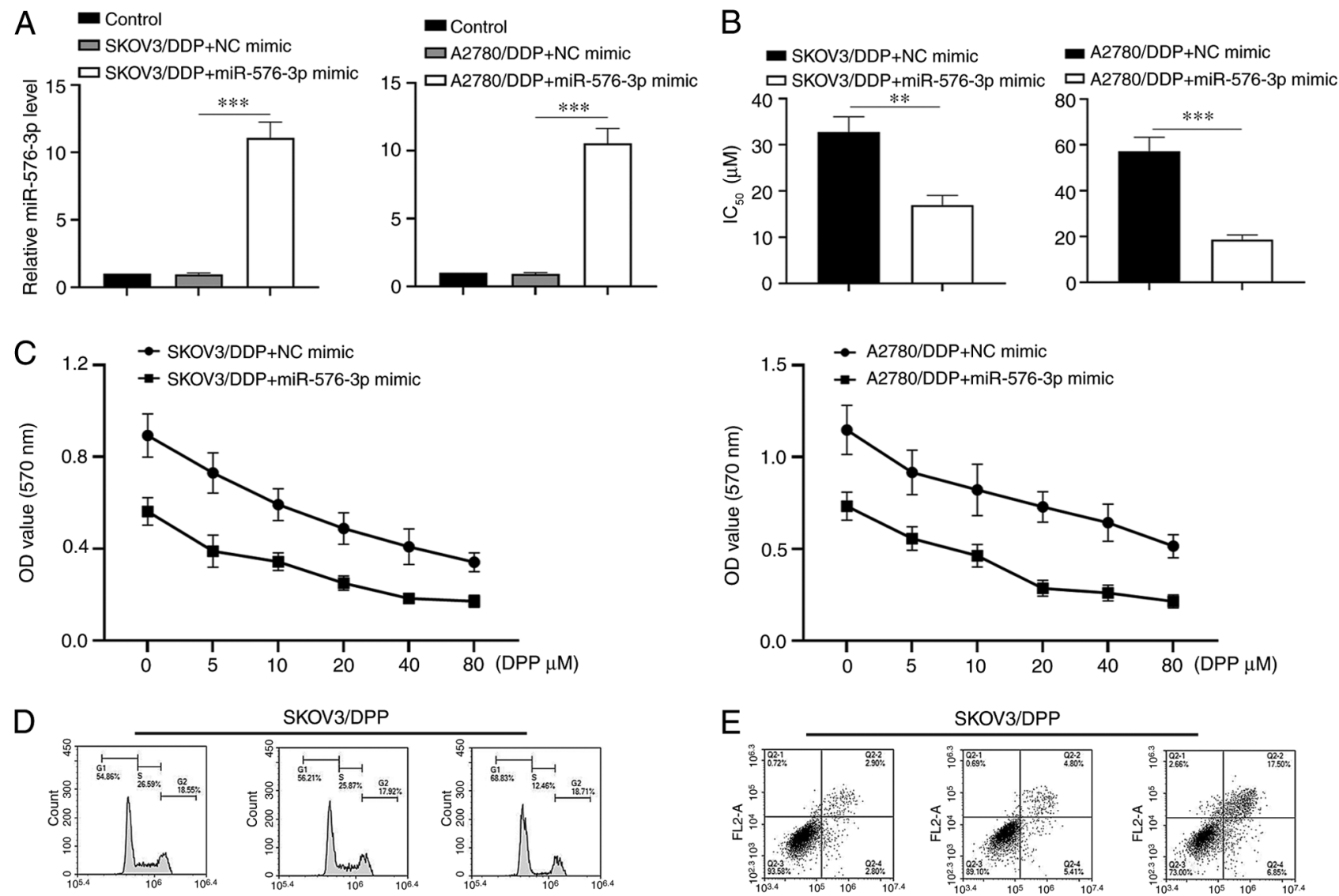

SKOV3/DPP

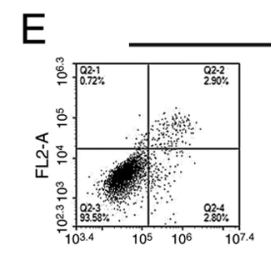

SKOV3/DPP
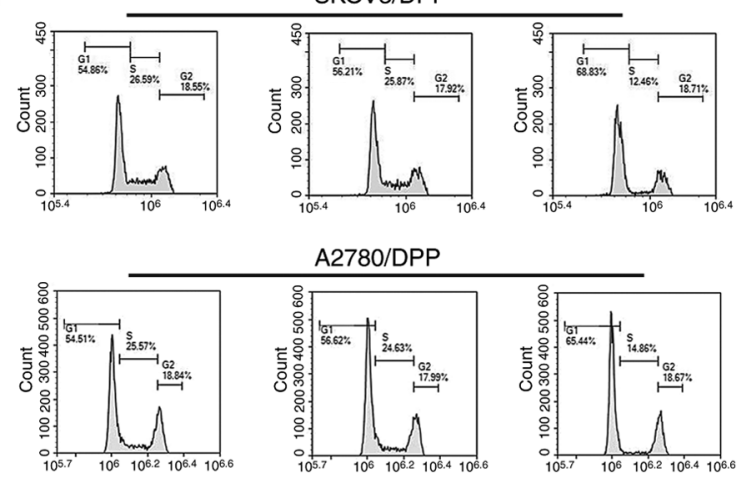

A2780/DPP

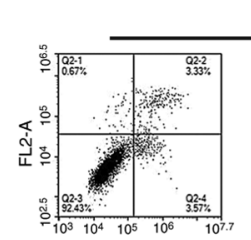

NC mimic
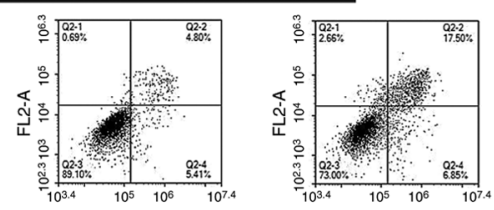

A2780/DPP

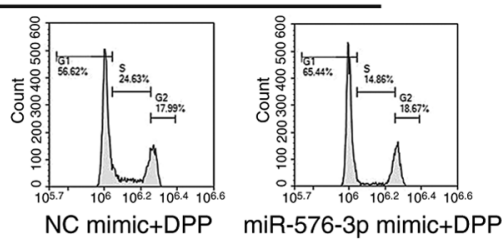

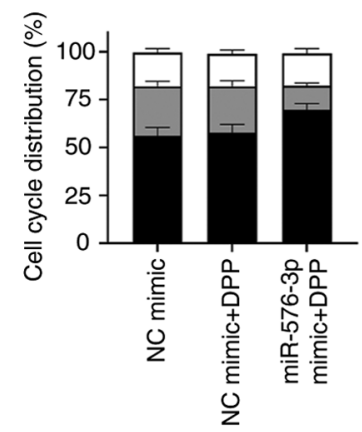

SKOV3/DPP

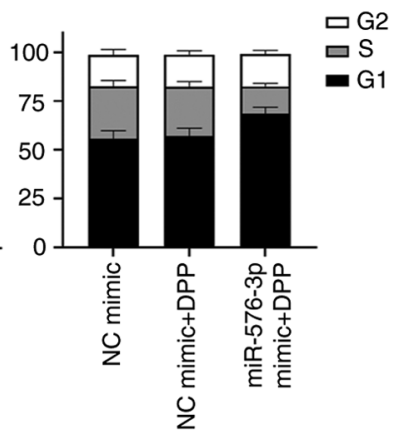

A2780/DPP

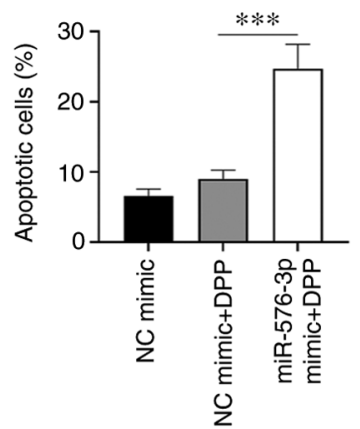

SKOV3/DPP

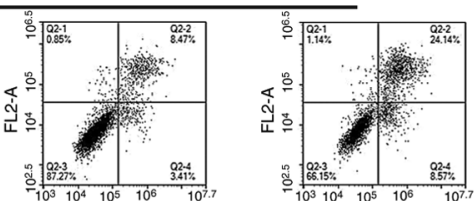

NC mimic+DPP miR-576-3p mimic+DPP

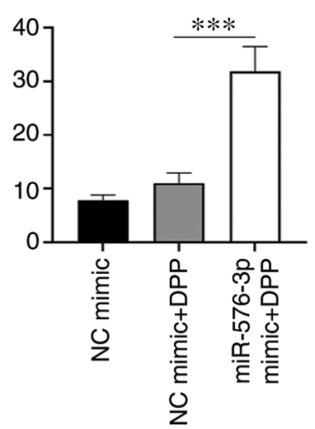

A2780/DPP

Figure 2. miR-576-3p overexpression increases DDP sensitivity of DDP-resistant ovarian cancer cells in vitro. (A) The expression levels of miR-576-3p in SKOV3/DDP and A2780/DDP cells transfected with miR-576-3p mimic and its NC. (B) IC $_{50}$ values of DDP in SKOV3/DDP and A2780/DDP cells transfected with miR-576-3p mimic and NC. (C) Effects of miR-576-3p overexpression on viability of SKOV3/DDP and A2780/DDP cells treated with gradually increasing concentrations of DDP. Effects of miR-576-3p overexpression on (D) cell cycle progression and (E) apoptosis in SKOV3/DDP and A2780/DDP cells treated with DDP. $n=3 .{ }^{* * *} \mathrm{P}<0.01$ and ${ }^{* * * *} \mathrm{P}<0.001$. miR, microRNA; DDP, cisplatin; NC, negative control.

reduced in SKOV3/DDP and A2780/DDP cells following miR-576-3p mimic transfection, but were significantly increased by transfection with a miR-576-3p inhibitor (Figs. 4C-F and S1B). Therefore, miR-576-3p overexpression may regulate cell viability and apoptosis via targeting PD-L1 and cyclin D1.
miR-576-3p overexpression enhances the efficacy of DDP in inhibiting ovarian tumor growth in vivo. The effects of miR-576-3p on DDP resistance in ovarian cancer cells were also investigated using a xenograft tumor model. The results demonstrated that DDP treatment inhibited tumor growth in the nude mice injected with miR-576-3p 

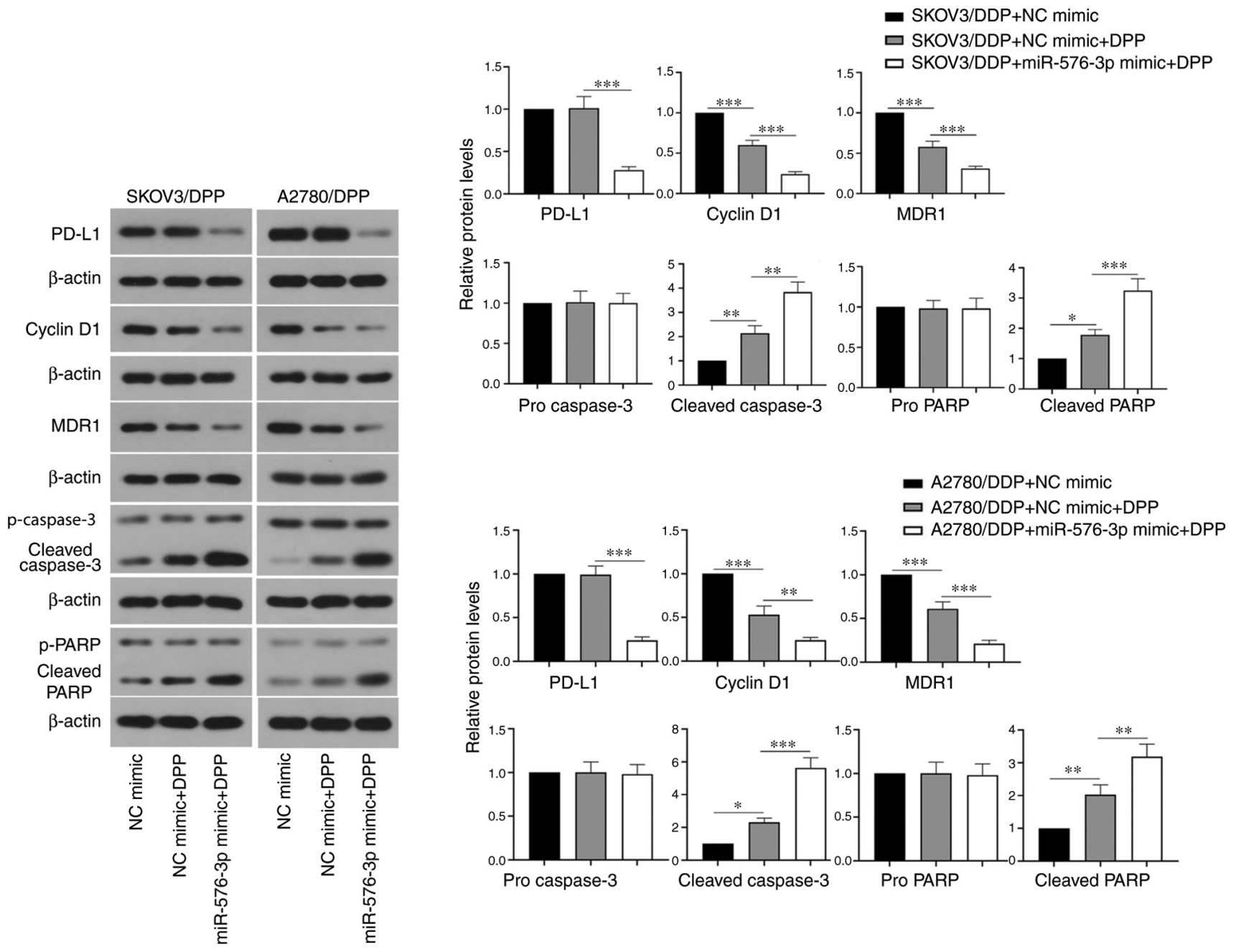

Figure 3. Effects of miR-576-3p overexpression on the protein expression levels of PD-L1, cyclin D1, MDR1, cleaved/pro caspase-3 and cleaved/pro PARP in SKOV3/DDP and A2780/DDP cells treated with DDP. $n=3$. ${ }^{*} \mathrm{P}<0.05,{ }^{* *} \mathrm{P}<0.01$ and ${ }^{* * * *} \mathrm{P}<0.001$. miR, microRNA; PD-L1, programmed death-ligand 1; DDP, cisplatin; MDR1, efflux pump multidrug resistance protein 1; PARP, poly(ADP-ribose) polymerase; NC, negative control.

overexpressing-SKOV3/DDP cells compared with the control group (Fig. 5A and B). The mRNA expression level of miR-576-3p was upregulated in miR-576-3p-overexpressing ovarian tumor tissues (Fig. 5C). Western blotting demonstrated that the protein expression levels of PD-L1, cyclin D1 and MDR1 were downregulated, whereas the cleaved/pro caspase-3 ratio and cleaved/pro PARP ratio were upregulated in miR-576-3p-overexpressing tumor tissues treated with DDP (Fig. 5D). Overall, miR-576-3p overexpression enhanced DDP sensitivity of DDP-resistant ovarian cancer cells in vivo.

\section{Discussion}

Ovarian cancer is one of the most malignant tumors of the female reproductive system. With the widespread application of chemotherapeutic drugs, patients with ovarian cancer may develop drug resistance that adversely affects their prognosis. Hence, it is crucial to identify methods that enhance the sensitivity of ovarian cancer cells to DPP. Various studies have indicated that miRNAs have a crucial role in cancer progression and chemosensitivity due to their important regulatory functions of targets at both the transcriptional and post-transcriptional levels. The findings of the present study suggested that miR-576-3p was downregulated in DDP-resistant ovarian cancer cells, and miR-576-3p overexpression may enhance DDP sensitivity of ovarian cancer cells via targeting PD-L1 and cyclin D1.

It was observed that DDP-resistant ovarian cancer cells SKOV3/DDP and A2780/DDP had a higher $\mathrm{IC}_{50}$ value compared with SKOV3 and A2780 cells, indicating that SKOV3 and A2780 cells were more sensitive to DDP compared with SKOV3/DDP and A2780/DDP cells. Furthermore, miR-576-3p overexpression significantly decreased the $\mathrm{IC}_{50}$ value of DDP-resistant ovarian cancer cells, indicating that miR-576-3p overexpression increased the sensitivity of DDP-resistant ovarian cancer cells to DDP. It was previously demonstrated that miR-576-3p was downregulated in non-melanoma skin cancer, lung adenocarcinoma and bladder cancer (9-11). The downregulation of miR-576-3p was found to be associated with the chemosensitivity of human teratoma and breast cancer cells $(12,13)$. The present study demonstrated that miR-576-3p overexpression inhibited viability and promoted apoptosis of DDP-resistant ovarian cancer cells. A previous study reported that the antitumor effects of DDP on drug-resistant ovarian cancer cells may be enhanced by induction of apoptosis and inhibition of cell migration and invasion (27). Therefore, 
A

\begin{tabular}{|c|c|}
\hline PD-L1 3'-UTR wt & $\begin{array}{l}5^{\prime} \text {...GCUUAAUGAUUUGCUCACAUCUA....3' } \\
\text { | | | | | | | }\end{array}$ \\
\hline hsa-miR-576-3p & $3^{\prime} \ldots C U A A G G U U A A A A A G G U G U A G A A \ldots 5^{\prime}$ \\
\hline PD-L1 3'-UTR mut & $5^{\prime} \ldots$ GCUUAAUGAUUUGCUGUGUAGAA $\ldots 3^{\prime}$ \\
\hline Cyclin D1 3'-UTR wt & $5^{\prime} \ldots$ AAAAUAGACAAUUUGCACAUCUU...3' \\
\hline hsa-miR-576-3p & 3'...CUAAGGUUAAAAAGGUGUAGAA ...5' \\
\hline Cyclin D1 3'-UTR mut & $5^{\prime} \ldots$ AAAAUAGACAAUUUGGUGUAGAU....3' \\
\hline
\end{tabular}

B

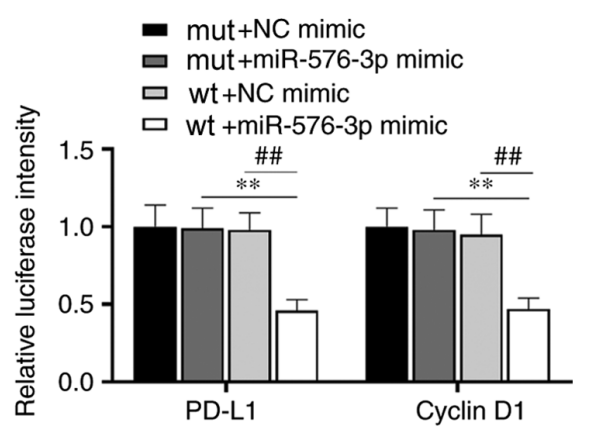

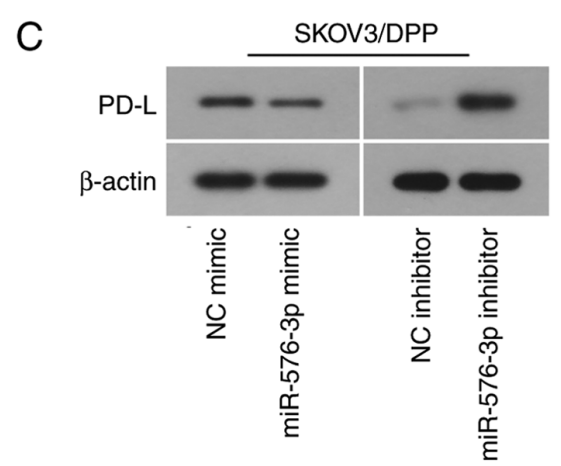

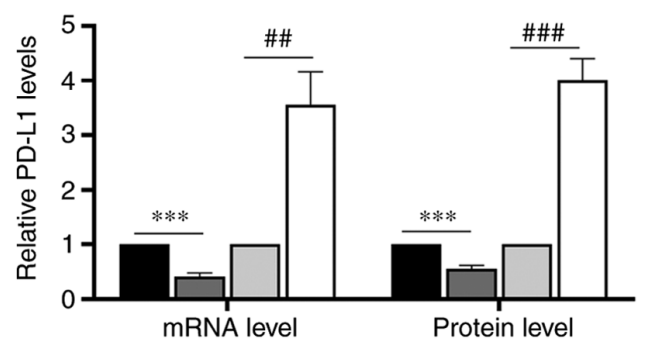

E
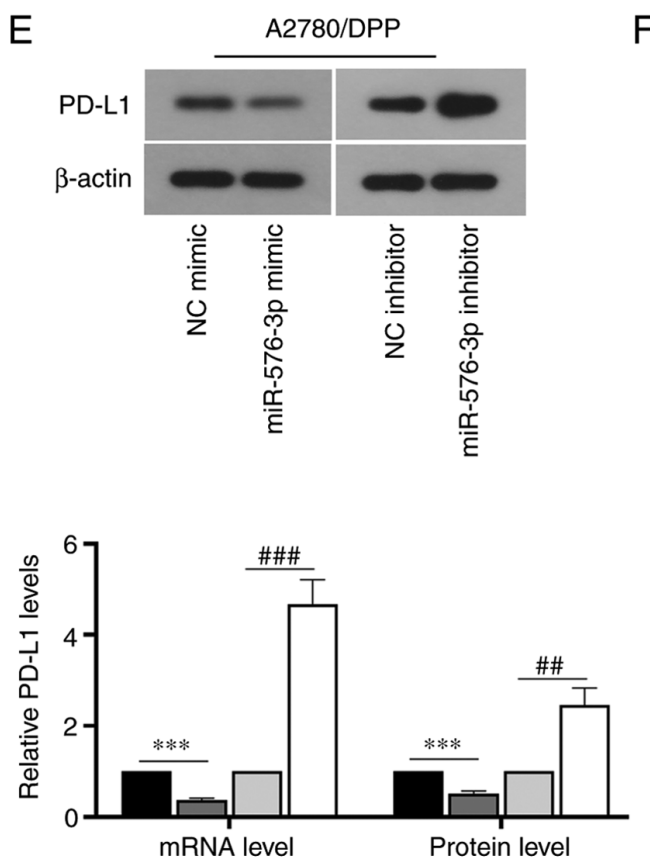

D

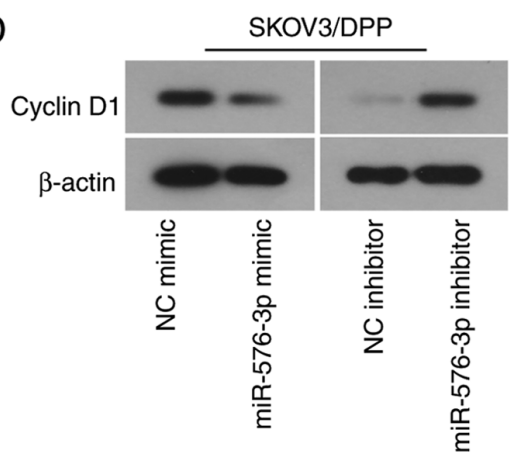

- SKOV3/DDP+NC mimic $\square$ SKOV3/DDP+miR-576-3p mimic $\square$ SKOV3/DDP+NC inhibitor $\square$ SKOV3/DDP+miR-576-3p inhibitor

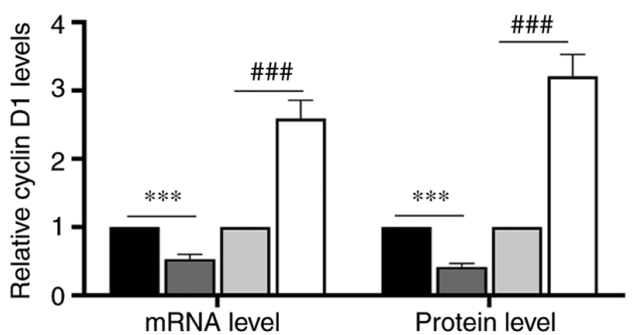

F
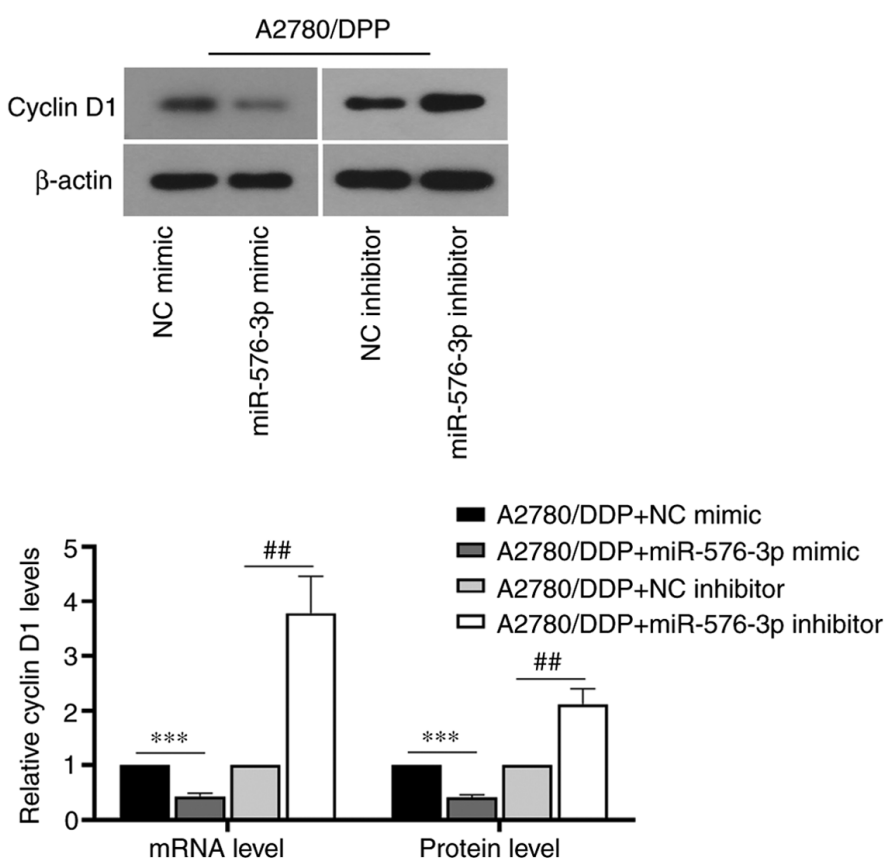

Figure 4. miR-576-3p directly targets PD-L1 and cyclin D1. (A) Binding sites of miR-576-3p to the 3'-UTR of PD-L1 and cyclin D1. (B) Luciferase activity was measured by using a dual-luciferase assay to validate the targeting relationship between miR-576-3p, PD-L1 and cyclin D1. (C-F) mRNA and protein expression levels of PD-L1 and cyclin D1 in SKOV3/DDP and A2780/DDP cells transfected with miR-576-3p mimic or inhibitor. $\mathrm{n}=3$. ${ }^{* *} \mathrm{P}<0.01$ and ${ }^{* * *} \mathrm{P}<0.001$; ${ }^{\# \#} \mathrm{P}<0.01$ and ${ }^{\# \#} \mathrm{P}<0.001$. miR, microRNA; PD-L1, programmed death-ligand 1; DDP, cisplatin; 3'-UTR, 3'-untranslated region; wt, wild-type; mut, mutant; $\mathrm{NC}$, negative control. 

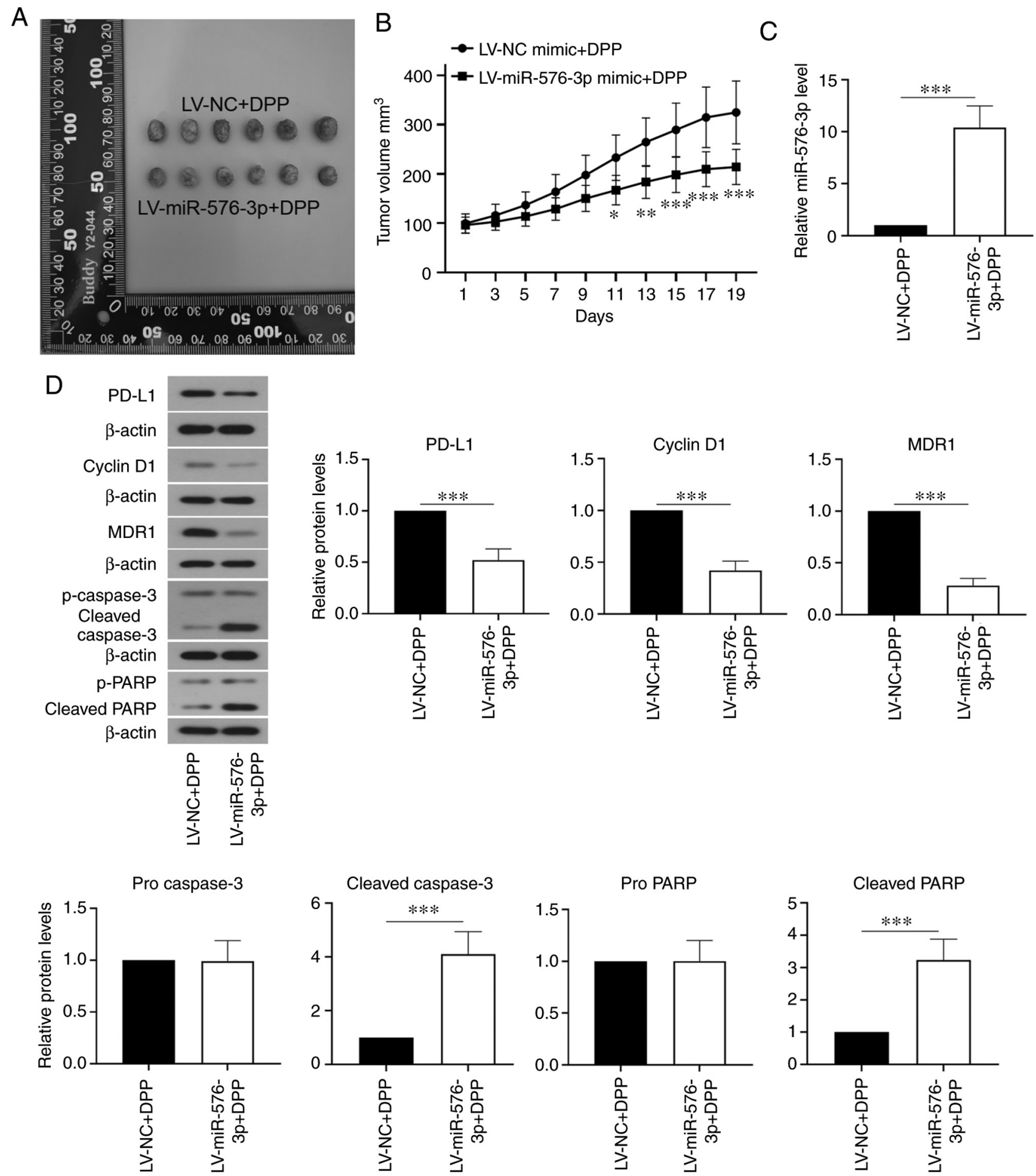

Figure 5. miR-576-3p overexpression enhances the efficacy of DDP in inhibiting ovarian tumor growth in vivo. (A and B) Changes in the tumor volume in nude mice injected with miR-576-3p-overexpressing SKOV3 cells treated with DDP. (C) Effects of miR-576-3p upregulation on mRNA expression levels of miR-576-3p and (D) the protein expression levels of PD-L1, cyclin D1, MDR1, cleaved/pro caspase-3 and cleaved/pro PARP in tumor tissues. $\mathrm{n}=6$. " $\mathrm{P}<0.05$, ${ }^{* *} \mathrm{P}<0.01$ and ${ }^{* * *} \mathrm{P}<0.001$. miR, microRNA; PD-L1, programmed death-ligand 1; DDP, cisplatin; MDR1, efflux pump multidrug resistance protein 1; PARP, poly(ADP-ribose) polymerase; LV, lentivirus; $\mathrm{NC}$, negative control.

the effects of miR-576-3p overexpression on promoting the sensitivity of ovarian cancer cells to DDP may be achieved by inhibiting viability and promoting apoptosis in DDP-resistant ovarian cancer cells.

In the present study, upregulation of PD-L1, MDR1 and cyclin D1 was observed in DDP-resistant ovarian cancer cells, whereas miR-576-3p overexpression reduced the expression levels of PD-L1, MDR1 and cyclin D1. MDR1 is an ATP-binding cassette transporter that acts as an energy-dependent efflux pump for multiple drugs and is responsible for reducing drug accumulation in cells, ultimately causing drug resistance (28). MDR1 efflux is considered to be the primary mechanism of ovarian cancer resistance to taxanes (29). MDR1 overexpression reduced the sensitivity 
of ovarian cancer cells to cisplatin and paclitaxel, whereas MDR1 knockdown increased chemosensitivity to vincristine, paclitaxel and docetaxel $(30,31)$. PD-L1 is an antitumor immune negative regulator. During cancer development, the increased binding of PD-L1 to the receptor PD-1 on T cells causes T-cell dysfunction, thereby preventing cytotoxic $\mathrm{T}$ cells from effectively targeting tumor cells, leading to tumor cell survival (32). Our previous research found that PD-L1 was highly expressed in DDP-resistant ovarian cancer cells, and PD-L1 silencing decreased this DDP resistance (19). Clinical studies have demonstrated that PD-L1 may be a potential mechanism used by cancer cells to evade immune defense, and inhibiting the interaction between PD-1 and PD-L1 may enhance the $\mathrm{T}$ cell response and mediate antitumor activity (33). Currently, PD-L1 inhibitors and anti-PD-L1 antibodies developed for PD-L1 have been successful in clinical trials of cancer therapy (34). It was reported that the combined administration of anti-PD-L1 antibodies and DDP significantly reduced tumor growth compared with single-agent treatments and controls (35). These findings indicated a key role of PD-L1 in regulating DDP resistance of cancer cells. The survival and proliferation of cancer cells require the expression of cyclin D1. Cyclin D1 acts as an allosteric modulator of cellular cyclin-dependent kinase 4 and 6 to regulate the transition of the cell cycle from the G1 to the S phase (36). Cyclin D1 overexpression was found to be associated with a high risk of recurrence of invasive serous ovarian cancer and poor response to first-line chemotherapy (37). Consistent with these previous findings, the results of the present study suggested that miR-576-3p overexpression increased the chemosensitivity of ovarian cancer cells to DDP via regulating PD-L1, MDR1 and cyclin D1 levels. Furthermore, in vivo studies suggested that miR-576-3p overexpression inhibited tumorigenesis and downregulated the expression of PD-L1, MDR1 and cyclin D1 in tumor tissues, which was consistent with the in vitro findings.

In bladder cancer, a targeting relationship between miR-576-3p and cyclin D1 has already been identified (38), and the present study also further confirmed this targeting relationship. Additionally, it was demonstrated that PD-L1 is one of the targets of miR-576-3p. However, to the best of our knowledge, there is no evidence of miR-576-3p targeting and regulating the expression of MDR1. The present study demonstrated that miR-576-3p may indirectly regulate the expression of MDR1 via PD-L1 and cyclin D1. A previous study demonstrated that the PD-1/PD-L1 interaction may upregulate the expression of MDR1 in breast cancer cells (39). In addition, knockdown of cyclin D1 significantly reduced the expression of MDR1 in human glioblastoma (40). Therefore, the effect of miR-576-3p overexpression on the chemosensitivity of ovarian cancer cells may be achieved by targeting PD-L1 and cyclin D1. Furthermore, it was previously reported that PD-L1 silencing promoted apoptosis of DDP-resistant ovarian cancer cells and inhibited cell proliferation, whereas PD-L1 overexpression inhibited apoptosis and promoted cell proliferation (19). DDP inhibited the proliferation of human epithelial ovarian cancer cells and promoted apoptosis via the inhibition of cyclin D1, and the overexpression of cyclin D1 increased the proliferative ability of epithelial ovarian cancer cells $(41,42)$. Collectively, these findings demonstrated that miR-576-3p overexpression may promote apoptosis and inhibit the viability of ovarian cancer cells via decreasing PD-L1 and cyclin D1 expression.

However, a limitation of the present study is that there were only six mice in each group for the animal experiments. Moreover, further studies are needed to explore whether miR-576-3p can regulate cisplatin sensitivity of ovarian cancer cells by directly regulating PD-L1 and cyclin D1 in vivo.

In conclusion, previous findings have indicated that both PD-L1 and cyclin D1 are important in the modulation of ovarian tumor progression. Combined with the findings of the present study, it was shown that that miR-576-3p played a key role in ovarian cancer development and DDP resistance via negatively regulating PD-L1 and cyclin D1 expression.

\section{Acknowledgements}

Not applicable.

Funding

No funding was received.

\section{Availability of data and materials}

The datasets used and/or analyzed during the current study are available from the corresponding author on reasonable request.

\section{Authors' contributions}

$\mathrm{YZ}$ and $\mathrm{WZ}$ performed the experiments, contributed to data analysis and made substantial contributions to the conception and design. QT, JL and SW contributed to data analysis and the experimental materials. CX made substantial contributions to the conception and design of the work, gave final approval of the version to be published, agreed to be accountable for all aspects of the work in ensuring that questions related to the accuracy or integrity of any part of the work are appropriately investigated and resolved, drafted the manuscript and critically revised the manuscript for important intellectual content. All authors read and approved the final manuscript.

\section{Ethics approval and consent to participate}

The present study was approved by the Institutional Animal Care and Use Committee of Affiliated Yantai Yuhuangding Hospital (approval no. 2018137; Shandong, China) and performed according to the Guidelines for the Care and Use of Laboratory Animals.

\section{Patient consent for publication}

Not applicable.

\section{Competing interests}

The authors declare that they have no competing interests. 


\section{References}

1. Torre LA, Bray F, Siegel RL, Ferlay J, Lortet-Tieulent J and Jemal A Global cancer statistics, 2012. CA Cancer J Clin 65: 87-108, 2015.

2. Bray F, Ferlay J, Soerjomataram I, Siegel RL, Torre LA and Jemal A: Global cancer statistics 2018: GLOBOCAN estimates of incidence and mortality worldwide for 36 cancers in 185 countries. CA Cancer J Clin 68: 394-424, 2018.

3. SalehiF, Dunfield L, Phillips KP, Krewski D and Vanderhyden BC: Risk factors for ovarian cancer: An overview with emphasis on hormonal factors. J Toxicol Environ Health B Crit Rev 11: 301-321, 2008.

4. Bristow RE and Chi DS: Platinum-based neoadjuvant chemotherapy and interval surgical cytoreduction for advanced ovarian cancer: A meta-analysis. Gynecol Oncol 103: 1070-1076, 2006.

5. Pignata S, C Cecere S, Du Bois A, Harter P and Heitz F: Treatment of recurrent ovarian cancer. Ann Oncol 28 (Suppl 8): viii51-viii6, 2017.

6. Bookman MA: Optimal primary therapy of ovarian cancer. Ann Oncol 27 (Suppl 1): i58-i62, 2016.

7. Jayson GC, Kohn EC, Kitchener HC and Ledermann JA: Ovarian cancer. Lancet 384: 1376-1388, 2014.

8. Hayes J, Peruzzi PP and Lawler S: MicroRNAs in cancer: Biomarkers, functions and therapy. Trends Mol Med 20: 460-469, 2014.

9. Balci S, Ayaz L, Gorur A, Yildirim Yaroglu H, Akbayir S, Dogruer Unal N, Bulut B, Tursen U and Tamer L: microRNA profiling for early detection of nonmelanoma skin cancer. Clin Exp Dermatol 41: 346-351, 2016.

10. Greenawalt EJ, Edmonds MD, Jain N, Adams CM, Mitra R and Eischen CM: Targeting of SGK1 by miR-576-3p Inhibits Lung Adenocarcinoma Migration and Invasion. Mol Cancer Res 17: 289-298, 2019.

11. Meng FM, Meng FM and Song XL: MiR-576-3p is a novel marker correlated with poor clinical outcome in bladder cancer. Eur Rev Med Pharmacol Sci 21: 973-977, 2017.

12. Port M, Glaesener S, Ruf C, Riecke A, Bokemeyer C, Meineke V, Honecker F and Abend M: Micro-RNA expression in cisplatin resistant germ cell tumor cell lines. Mol Cancer 10: 52, 2011.

13. Lv J, Xia K, Xu P, Sun E, Ma J, Gao S, Zhou Q, Zhang M, Wang F, Chen F, et al: miRNA expression patterns in chemoresistant breast cancer tissues. Biomed Pharmacother 68: 935-942, 2014

14. Wang X, Wu WKK, Gao J, Li Z, Dong B, Lin X, Li Y, Li Y, Gong J, Qi C, et al: Autophagy inhibition enhances PD-L1 expression in gastric cancer. J Exp Clin Cancer Res 38: 140, 2019

15. Wyss J, Dislich B, Koelzer VH, Galván JA, Dawson H, Hädrich M, Inderbitzin D, Lugli A, Zlobec I and Berger MD: Stromal PD-1/PD-L1 expression predicts outcome in colon cancer patients. Clin Colorectal Cancer 18: e20-e38, 2019.

16. Rojkó L, Reiniger L, Téglási V, Fábián K, Pipek O, Vágvölgyi A, Agócs L, Fillinger J, Kajdácsi Z, Tímár J, et al: Chemotherapy treatment is associated with altered PD-L1 expression in lung cancer patients. J Cancer Res Clin Oncol 144: 1219-1226, 2018.

17. Cai J, Qi Q, Qian X, Han J, Zhu X, Zhang Q and Xia R: The role of PD-1/PD-L1 axis and macrophage in the progression and treatment of cancer. J Cancer Res Clin Oncol 145: 1377-1385, 2019.

18. Jiang Z, Yang Y, Yang Y, Zhang Y, Yue Z, Pan Z and Ren X: Ginsenoside $\operatorname{Rg} 3$ attenuates cisplatin resistance in lung cancer by downregulating PD-L1 and resuming immune. Biomed Pharmacother 96: 378-383, 2017

19. Zuo Y, Zheng W, Liu J, Tang Q, Wang SS and Yang XS: MiR-34a-5p/PD-L1 axis regulates cisplatin chemoresistance of ovarian cancer cells. Neoplasma 67: 93-101, 2019.

20. Sherr CJ: G1 phase progression: Cycling on cue. Cell 79: 551-555, 1994.

21. Hu J, Fang Y, Cao Y, Qin R and Chen Q: miR-449a Regulates proliferation and chemosensitivity to cisplatin by targeting cyclin D1 and BCL2 in SGC7901 cells. Dig Dis Sci 59: 336-345, 2014.

22. BiliranHJr, Wang Y,Banerjee S, XuH,Heng H,Thakur A,Bollig A, Sarkar FH and Liao JD: Overexpression of cyclin D1 promotes tumor cell growth and confers resistance to cisplatin-mediated apoptosis in an elastase-myc transgene-expressing pancreatic tumor cell line. Clin Cancer Res 11: 6075-6086, 2005.
23. Liu R, Guo H and Lu S: MiR-335-5p restores cisplatin sensitivity in ovarian cancer cells through targeting BCL2L2. Cancer Med 7: 4598-4609, 2018.

24. Zou J, Liu L, Wang Q, Yin F, Yang Z, Zhang W and Li L: Downregulation of miR-429 contributes to the development of drug resistance in epithelial ovarian cancer by targeting ZEB1. Am J Transl Res 9: 1357-1368, 2017.

25. Livak KJ and Schmittgen TD: Analysis of relative gene expression data using real-time quantitative PCR and the 2(-Delta Delta C(T)) method. Methods 25: 402-408, 2001

26. National Research Council (US) Committee for the Update of the Guide for the Care and Use of Laboratory Animals: Guide for the Care and Use of Laboratory Animals. 8th edition. National Academies Press (US), Washington, DC, 2011.

27. Wang H, Luo Y, Qiao T, Wu Z and Huang Z: Luteolin sensitizes the antitumor effect of cisplatin in drug-resistant ovarian cancer via induction of apoptosis and inhibition of cell migration and invasion. J Ovarian Res 11: 93, 2018.

28. Joncourt F, Buser K, Altermatt H, Bacchi M, Oberli A and Cerny T: Multiple drug resistance parameter expression in ovarian cancer. Gynecol Oncol 70: 176-182, 1998.

29. Kavallaris M, Kuo DY, Burkhart CA, Regl DL, Norris MD, Haber $\mathrm{M}$ and Horwitz SB: Taxol-resistant epithelial ovarian tumors are associated with altered expression of specific beta-tubulin isotypes. J Clin Invest 100: 1282-1293, 1997.

30. Wu DD, Li XS, Meng XN, Yan J and Zong ZH: MicroRNA-873 mediates multidrug resistance in ovarian cancer cells by targeting ABCB1. Tumour Biol 37: 10499-10506, 2016

31. Zhang H, Wang J, Cai K, Jiang L, Zhou D, Yang C, Chen J, Chen D and Dou J: Downregulation of gene MDR1 by shRNA to reverse multidrug-resistance of ovarian cancer A2780 cells. J Cancer Res Ther 8: 226-231, 2012.

32. Zhang J, Dang F, Ren J and Wei W: Biochemical aspects of PD-L1 regulation in cancer immunotherapy. Trends Biochem Sci 43: 1014-1032, 2018

33. Iwai Y, Ishida M, Tanaka Y, Okazaki T, Honjo T and Minato N: Involvement of PD-L1 on tumor cells in the escape from host immune system and tumor immunotherapy by PD-L1 blockade. Proc Natl Acad Sci USA 99: 12293-12297, 2002.

34. Hamanishi J, Mandai M, Matsumura N, Abiko K, Baba T and Konishi I: PD-1/PD-L1 blockade in cancer treatment: Perspectives and issues. Int J Clin Oncol 21: 462-473, 2016.

35. Fournel L, Wu Z, Stadler N, Damotte D, Lococo F, Boulle G, Ségal-Bendirdjian E, Bobbio A, Icard P, Trédaniel J, et al: Cisplatin increases PD-L1 expression and optimizes immune check-point blockade in non-small cell lung cancer. Cancer Lett 464: 5-14, 2019.

36. Qie S and Diehl JA: Cyclin D1, cancer progression, and opportunities in cancer treatment. J Mol Med (Berl) 94: 1313-1326, 2016.

37. Abdelrahman AE, Fathy A, Elsebai EA, Nawar N and Etman WM Prognostic impact of Apaf-1, Cyclin D1, and AQP-5 in serous ovarian carcinoma treated with the first-line chemotherapy. Ann Diagn Pathol 35: 27-37, 2018

38. Liang Z, Li S, Xu X, Xu X, Wang X, Wu J, Zhu Y, Hu Z, Lin Y, Mao Y, et al: MicroRNA-576-3p inhibits proliferation in bladder cancer cells by targeting cyclin D1. Mol Cells 38: 130-137, 2015.

39. Liu S, Chen S, Yuan W, Wang H, Chen K, Li D and Li D: PD-1/PD-L1 interaction up-regulates MDR1/P-gp expression in breast cancer cells via PI3K/AKT and MAPK/ERK pathways. Oncotarget 8: 99901-99912, 2017.

40. Wang J, Wang Q, Cui Y, Liu ZY, Zhao W, Wang CL, Dong Y, Hou L, Hu G, Luo C, et al: Knockdown of cyclin D1 inhibits proliferation, induces apoptosis, and attenuates the invasive capacity of human glioblastoma cells. J Neurooncol 106: 473-484, 2012

41. Dai J, Wei RJ, Li R, Feng JB, Yu YL and Liu PS: A study of CCND1 with epithelial ovarian cancer cell proliferation and apoptosis. Eur Rev Med Pharmacol Sci 20: 4230-4235, 2016.

42. Xia B, Yang S, Liu T and Lou G: miR-211 suppresses epithelial ovarian cancer proliferation and cell-cycle progression by targeting Cyclin D1 and CDK6. Mol Cancer 14: 57, 2015. 